\title{
A Report on Use of 10\% Lidocaine Neurolysis for Chronic Refractory Supraorbital Neuralgia
}

\author{
Gaurav Chauhan, MD, Daanish Khaja, MD, Farayi Mbuvah, MD, and Aman Upadhyay, MD
}

We present a case report of a 50-year-old female with a past medical history of chronic headaches diagnosed as supraorbital neuralgia. Her headaches had been refractory to conventional analgesic medications and she had received multiple supraorbital nerve blocks which gave her temporary relief of the headaches lasting up to 2 weeks. The patient also received chemical neurolysis of the supraorbital nerve bilaterally with $10 \%$ Lidocaine bilaterally, under fluoroscopic guidance in the pain clinic. The patient reported positive outcome following the block with significant pain control for up to 6-7 months postprocedure. The authors conclude that in a patient with a refractory headache due to supraorbital neuralgia, a $10 \%$ lidocaine,injection can be an efficacious alternative to radiofrequency ablation as it yields optimum symptom control for up to 6 - 7 months and is a relatively easy procedure to perform. Furthermore, the procedure is not associated with significant side effects and with reversibility of induced sensory functional deficits, offering an advantage over radiofrequency ablation.

Key words: Lidocaine, supraorbital neuralgia, neurolysis, nerve blocks, flouroscopy
The supraorbital nerve is a purely sensory branch of the ophthalmic division of the trigeminal nerve. It innervates the skin of the forehead, eyelid, conjunctiva and frontal sinus. As the nerve exits the supraorbital notch its becomes more superficial and becomes more vulnerable to trauma and compression $(1,2)$. Supraorbital neuralgia, also known as epicranial neuralgia, is an uncommon pain syndrome with a prevalence of $0.65 \%$ in the adult population (3). The most common etiology is trauma to the supraorbital nerve and it is the leading cause of supraorbital neuralgia in males. An idiopathic or non-traumatic origin is reported in only $0.15 \%$ cases of supraorbital neuralgia and is usually the predominant cause of headaches

From : Henry Ford Health System, Detroit, MI

Author for correspondence: Gaurav Chauhan, M.D.

Address: Department of Anesthesiology, Pain Management and Perioperative Medicine, Henry Ford Health System, Apt 1607, 1350 W. Bethune St., Detroit, MI - 48202

E-mail: Gchauha1@hfhs.org in females. The pain associated with supraorbital neuralgia is usually insidious in onset. The median age of diagnosis is 50 years with diagnosis defined by the International Classification of Headache Disorders - II criterion (4). The differential diagnosis includes supratrochlear neuralgia, trigeminal neuralgia affecting the ophthalmic division, hemicrania continua, trigeminal autonomic cephalagias, etc. A presumptive diagnosis is made on clinical symptoms of intermittent or constant unilateral or bilateral pain in the medial part of the forehead. The most common sign is pain when pressure is applied over the supraorbital notch. A definitive diagnosis is clinched by the pain abolished by the supraorbital nerve block $(4,5)$. It is rare to have associated migrainous features like phono or photophobias, conjunctival injection or lacrimation. Due to neurogenic nature of pain, symptom control is challenging with multimodal analgesic therapies usually rendered ineffective $(4,6)$. We present a case of supraorbital neuralgia refractory to conventional multimodal therapy with analgesic medication supplemented with nerve blocks and 
will also discuss the reasons behind the therapeutic rationale employed by the authors.

\section{CLINICAL VIGNETTE}

We present a case report of a 50-year-old Caucasian female, who consented to this case report being published. The subject presented with a bilateral, intermittent, throbbing frontal headache that was more severe in the region of the supraorbital notch bilaterally and at the medial aspect of the forehead. The patient reported that the pain was intermittent in nature and variable in intensity, ranging from 2 to 8 out of 10 on the numeric rating scale for pain. The patient further stated that the pain was insidious in onset and the character of pain had remained unchanged over the last 3 years. The pain was not associated with any aura, phono/photophobias, feelings of nausea etc. She also reported that she underwent extensive neurological exams, blood tests and imaging of the brain which were all negative for any kind of acute or chronic pathology. She was diagnosed with supraorbital neuralgia, at an outside facility, based on the clinical signs of the pain getting worse on applying pressing on the supraorbital notch bilaterally and the fact that the pain was abolished by supraorbital nerve block. The patient underwent supraorbital nerve block procedures multiple times at an outside facility, with $0.5 \%$ bupivacaine solution, whenever she developed flare-up of her symptoms. She reported suboptimal pain control with non-steroidal anti-inflammatory (NSAIDs) therapy (acetaminophen, indomethacin, ibuprofen, etodolac, etc.), gabapentinoids and sumatriptan in the past, with supraorbital nerve blocks were able to provide appreciable pain relief ranging from 7-10 days only. The patient stated that her quality of life is being affected as she feels depressed and frequently develops episodes of anxiety and panic attacks during a flare-up of her chronic refractory headaches. The patient was adequately counseled, educated and was scheduled for a bilateral supraorbital nerve block with $10 \%$ lidocaine. The patient was provided with peri-procedural anxiolysis with 2 $\mathrm{mg}$ midazolam and $50 \mathrm{mcg}$ fentanyl, administered intravenously. In the procedure room, under all sterile precautions, the superior orbital ridge was palpated to locate the supraorbital foramen and the site was marked bilaterally. A 25-gauge needle was advanced till the periosteum was contacted and at that point, $0.1 \mathrm{~mL}$ of the contrast was injected, under fluoroscopic guidance, for confirmation of the needle placement and to rule out the vascular spread of the contrast dye (Fig. 1). After negative aspiration, $1 \mathrm{~mL}$ of $10 \%$ lidocaine was injected into the region of the supraorbital notch under fluoroscopic guidance. The procedure was repeated again for the other side. To prevent the swelling of the anesthetic into the upper eyelid, pressure was applied for 5 minutes, after each injection, at the upper orbital rim. The patient was observed for 120 minutes in the post-procedure area before discharge. The patient tolerated the procedure well and at 6 months follow-up, she reported that the pain was easily controlled with oral NSAIDs and she required less breakthrough medication. She also reported that she didn't develop any paresthesiae or any other sensory deficits over the injection site and didn't report any kind of side effects that could be attributed to the nerve block. She rescheduled and underwent bilateral supraorbital nerve block with $10 \%$ lidocaine at 7 months after the initial injection. The patient reported a similar nature of pain relief 2 months after the second procedure.

\section{DISCUSSION}

The proposed mechanism for a nerve block with lidocaine involves blockade of fast-acting sodium channels leading to a decrease in spontaneous and triggered after-discharges and a reduction in crossexcitation among neighboring afferent nerve fibers. This resultant electrochemical environment increases the threshold of ectopic impulse generation and decreases the afferent discharges from the nociceptors. The blockade of sodium channels, which creates in vivo conditions conducive for pain mitigation, is more persistent with $10 \%$ lidocaine (7). Furthermore, various studies employing animal and cell culture studies have confirmed transient neurotoxic effect, as evidenced by histological exam, on cells exposed to lidocaine concentrations greater than or equal to $8 \%(8,9)$. Other therapeutic options available to the authors, involved the use of continuous or pulsed radiofrequency ablation (RFA), which despite offering a similar duration of pain control, is frequently associated with post-denervation neuralgia (10). The other alternative was chemical neurolysis with phenol and alcohol, which was ruled out given the possibility of soft tissue necrosis and cosmetic disfigurement of 


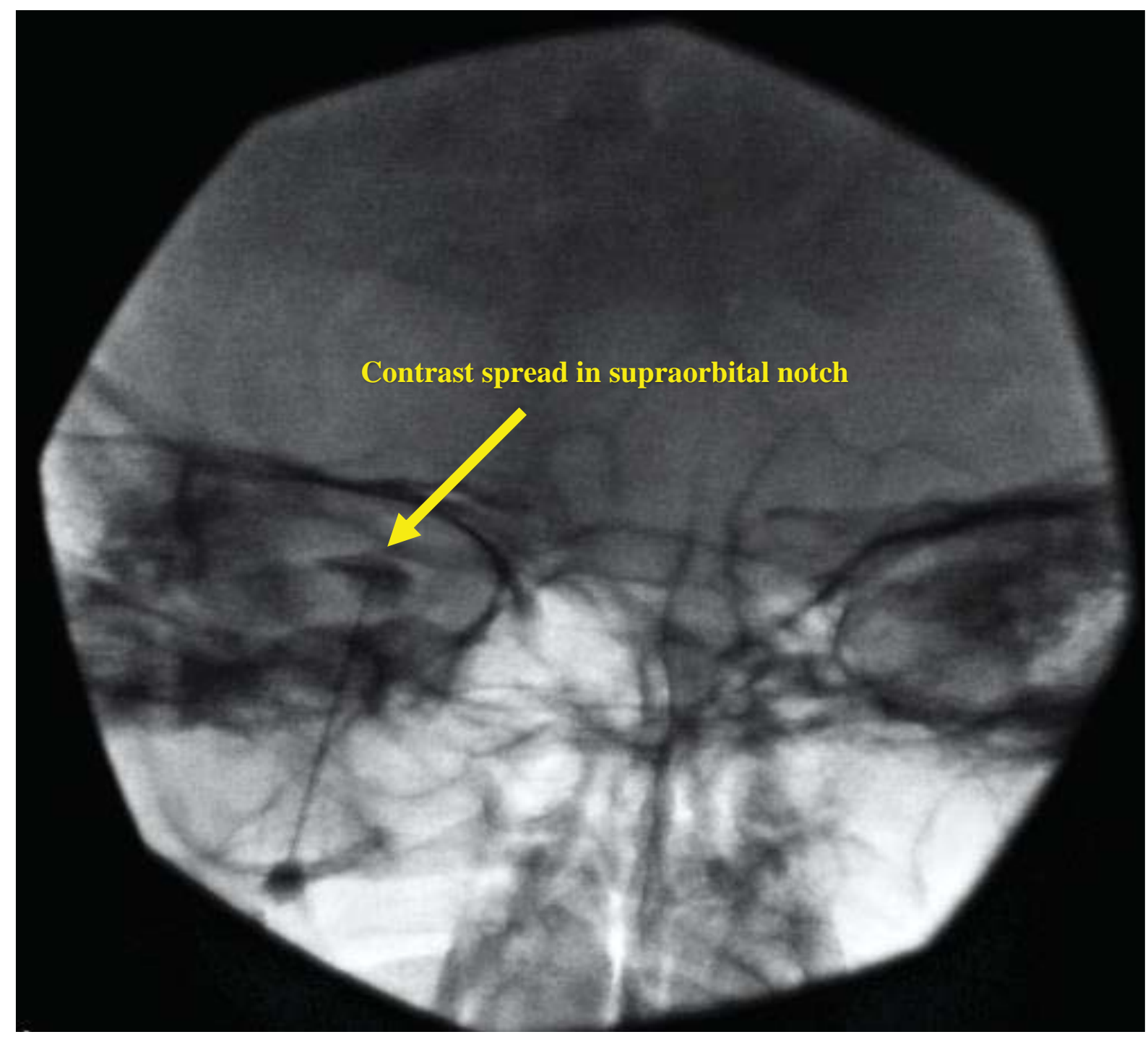

Fig. 1. Anterior-posterior fluoroscopic view of the face (left side) - needle with a little contrast to confirm proper location spread as well as ruling out vascular uptake.

the tissues surrounding the supraorbital nerve. Kim et al (11) in their retrospective review of 13 patients with migraine and occipital neuralgia that received greater occipital nerve block with $10 \%$ lidocaine, reported a significant reduction in visual analogue pain score for a mean duration of 148.05 days (standard deviation of 98.87 ) as compared to 6.33 days with $0.5 \%$ bupivacaine. Kim et al (11) concluded that $10 \%$ lidocaine, with comparable results and no reported side effects, can be used as a safe and effective alternative to radiofrequency ablation for neurolysis as far as symptom control for chronic neuropathic pain is concerned. Han et al (12) reported the clinical outcomes of trigeminal nerve block using $10 \%$ lidocaine and concluded that besides a relative simplicity inherent to the procedure, it is safe to perform and associated with complete reversibility of induced sensory deficits along with providing long duration of 
pain relief comparable to RFA. The concentration-dependent side effects associated with the use of $10 \%$ lidocaine for nerve blocks are transient neurologic symptoms, with incidence of $4 \%$ to $33 \%$, reported in current literature. Other adverse effects such as dizziness, blurred vision, profound hypertension, slurred speech, pseudo-seizures and seizures range from $0.1 \%$ to $9 \%$ and are proposed to be independent of the concentration or dose of the Lidocaine used (13). The rare side effects associated with the use of $10 \%$ lidocaine for supraorbital nerve block, limited to sporadic case reports, are ocular muscle necrosis leading to diplopia and ptosis $(14,15)$. For a positive outcome and to avoid inadvertent side effects, it is vital to employ risk reduction strategies at every step of this procedure. The authors first did a thorough review of the literature and provided optimum education and counseling to the patient in preparation for the procedure. On the day of the procedure, the drug was verified for use by 2 physicians and the subject was pre-medicated with midazolam to raise the seizure threshold. Fluoroscopy-guided precision along with the injection of radiopaque contrast was employed to avoid the inadvertent injection of drug

\section{REFERENCES}

1. Nanayakkara $D$, Manawaratne $R$, Sampath $H$, Vadysinghe $A$, Peiris R. Supraorbital nerve exits: positional variations and localization relative to surgical landmarks. Anatomy \& Cell Biology 2018; 51:19-24.

2. Shimizu S. Scalp neuralgia and headache elicited by cranial superficial anatomical causes: Supraorbital neuralgia, occipital neuralgia, and post-craniotomy headache. Rinsho Shinkeigaku 2014; 54:387-394.

3. Pareja JA, Caminero AB. Supraorbital neuralgia. Curr Pain Headache Rep 2006; 10:302-305.

4. Headache Classification Committee of the International Headache Society. Classification and diagnostic criteria for headache disorders, cranial neuralgias, and facial pain. 2nd edition. Suppl 1. Cephalalgia 2004; 24:9-160.

5. Sjaastad O, Stolt-Nielsen A, Pareja JA, Fredriksen TA, Vincent M. Supraorbital neuralgia. On the clinical manifestations and a possible therapeutic approach. Headache 1999; 39:204-212.

6. Gadient PM, Smith JH. The neuralgias: diagnosis and management. Curr Neurol Neurosci Rep 2014; 14:459.

7. Lavoie PA, Khazen T, Filion PR. Mechanisms of the inhibition of fast axonal transport by local anesthetics. Neuro-pharmacology 1989; 28:175-181.

8. Takenami T, Yagishita S, Asato F, Arai M, Hoka S. Intrathecal lidocaine causes posterior root axonal degeneration near entry into vascular and other tissue planes. Furthermore, the concentration of lidocaine $(1 \mathrm{~mL}$ bilaterally - 200 mg total dose) was kept below the recommended limits (maximum dose of $450 \mathrm{mg}$ for $90 \mathrm{Kg}$ subject) to avoid side effects.

\section{CONCLUSION}

In conclusion, bilateral supraorbital nerve blocks with $10 \%$ lidocaine, in a patient with a refractory headache due to supraorbital neuralgia, can be an efficacious alternative to RFA as it yields optimum symptom control for up to $6-7$ months and is relatively easier to perform. Furthermore, it is not associated with significant side effects and with reversibility of induced sensory functional deficits, which offers an advantage over RFA. It may be an attractive alternative for patients who have contraindications to RFA such as pacemaker/defibrillators or RFA cannot be performed due to technical issues placing the electrode. The conclusions are limited as they are based on clinical outcome of a single patient and a case series or a randomized, clinical trial should be designed to verify the reported observations. into the spinal cord in rats. Reg Anesth Pain Med 2002; 27:58-67.

9. Kim DD, Asif A, Kataria S. Presentation of neurolytic effect of $10 \%$ lidocaine after perineural ultrasound guided injection of a canine sciatic nerve: A pilot study. Korean J Pain 2016; 29:158163.

10. Gazelka HM, Knievel S, Mauck WD, Moeschler SM, Pingree MJ, Rho RH, Lamer TJ. Incidence of neuropathic pain after radiofrequency denervation of the third occipital nerve. J Pain Res 2014; 7:195-198.

11. Kim DD, Sibai N. Prolongation of greater occipital neural blockade with $10 \%$ lidocaine neurolysis: A case series of a new technique. J Pain Res 2016; 9:721-725.

12. Han KR, Kim C, Chae YJ, Kim DW. Efficacy and safety of high concentration lidocaine for trigeminal nerve block in patients with trigeminal neuralgia. Int J Clin Pract 2008; 62:248-254.

13. Yang S, Abrahams MS, Hurn PD, Grafe MR, Kirsch J. Local anesthetic Schwann cell toxicity is time and concentration dependent. Reg Anesth Pain Med 2011; 36:444-451.

14. Rainin EA, Carlson BM. Postoperative diplopia and ptosis. A clinical hypothesis based on the myotoxicity of local anesthetics. Arch Opthalmol 1985; 103:1337-1339.

15. Blum RA, Lim LT, Weir CR. Diplopia following sub-tenon's anaesthesia: An unusual complication. Int Ophthalmol 2012; 32:191-193. 